\title{
Semantic Analysis of Literary Vocabulary Based on Microsystem and Computer Aided Deep Research
}

\author{
Lixin $\mathrm{Li}^{1}$ and Liwen Cao $\mathbb{D}^{2}$ \\ ${ }^{1}$ Department of Foreign Languages, Xijing University, Xi'an 710123, Shaanxi, China \\ ${ }^{2}$ Department of Economics and Information Management, Shanghai University of Finance and Economics Zhejiang College, \\ Jinhua 321000, Zhejiang, China
}

Correspondence should be addressed to Liwen Cao; adarby4@mail.greenriver.edu

Received 26 June 2021; Revised 4 August 2021; Accepted 14 August 2021; Published 14 September 2021

Academic Editor: Sang-Bing Tsai

Copyright (C) 2021 Lixin Li and Liwen Cao. This is an open access article distributed under the Creative Commons Attribution License, which permits unrestricted use, distribution, and reproduction in any medium, provided the original work is properly cited.

\begin{abstract}
It has great advantages in data processing. Embedded microsystems are widely used in IoT devices because of their specific functions and hard decoding technology. This article adds a literary vocabulary semantic analysis model to the embedded microsystem to reduce power consumption and improve the accuracy and speed of the system. The main purpose of this paper is to improve the accuracy and speed of semantic analysis of literary vocabulary based on the embedded microsystem, combined with the design idea of Robot Process Automation (RPA) and adding CNN logic algorithm. In this paper, RPA Adam model is proposed. The RPA Adam model indicates that the vector in the vector contains not only the characteristics of its own node but also the characteristics of neighboring nodes. It is applied to graph convolution network of isomorphic network analysis and analyzes the types of devices that can be carried by embedded chips, and displays them with graphics. Through the results, we find that the error rate of the RPA Adam model is the same at different compression rates. Due to the different correlations between knowledge entities in different data sets, specifically, high frequency can maintain a low bit error rate of $10.79 \%$ when the compression rate is $4.85 \%$, but when the compression rate of high frequency is only $60.32 \%$, the error rate is as high as $11.26 \%$, while the compression rate of low frequency is $23.51 \%$ when the error rate is $9.65 \%$.
\end{abstract}

\section{Introduction}

Compared with other traditional processing technologies, embedded systems have advantages over integrated platforms in the data processing. Embedded microsystems generally use hard decoding to process data. Therefore, in the semantic analysis of literary vocabulary, a large number of data sets can be directly analyzed and output by hardware. At present, there are many types of research on the noise reduction of speech data, classifying the vocabulary of the literature.

On the processing of SQL data sets, code $f$ introduces bidirectional attention mechanism on the basis of sqlnet, which is different from the one-way calculation of column attention. It calculates forward and backward two rounds of attention for NLQ and column name, respectively, to capture the association between NLQ and column name and realize the mutual emphasis of two kinds of information [1]. In the $\mathrm{x}$-sql model proposed by Androutsopoulos et al.; firstly, mt-dnn is used to replace the best large used by sqlova in the selection of Bert, and a better generation effect is obtained [2]. The typesql model proposed after $K$ sets the preprocessing operation of NLQ annotation. Annotation types involve database elements, numbers, dates, and entity names [3]. Blunschi et al. tested RNN, CNN, and transformer in three SPARQL datasets [4]. In order to better adapt to the sample characteristics of the SPARC data set, Zenz et al. sequence model has done four attention designs [5]. Based on the graph model, Saha et al. interactively explore the communication behavior of mobile users from the perspective of ego network and propose an abnormal communication behavior analysis system egostellar [6]. 
Fei and Jagadish construct a Bayesian location reasoning framework, which can find abnormal large-scale aggregation events from large-scale communication data and further infer who participated in the event $[7,8]$. Different from anonymization, $\mathrm{Wu}$ et al. annotate the database column names or condition values (hereinafter referred to as database elements) mentioned in NLQ in the input sequence [9]. Zhang et al. proposed a visual analysis model of community and user behavior from the perspective of social and spatiotemporal characteristics of communication network users to support security departments to identify abnormal user events and abnormal group activities [10]. Chen et al. designed a visual analysis system called aureole to perceive the temporal and spatial distribution and utilization of word roots. The system uses the ring composition theory to let users focus on the region of interest without losing context information [11]. Bahdanau et al. proposed a novel systematic method to obtain travel information of lexical users from communication data [12]. The above research mainly focuses on semantic analysis in the field of communication. A variety of semantic analysis schemes are proposed on the basis of the neural network, but there are still great shortcomings in hardware decoding. The key is that the algorithm cannot be well connected with the interface of the hardware decoder [13].

In this paper, based on the embedded microsystem, combined with the design idea of robot process automation (RPA), a CNN logic algorithm is added to improve the accuracy and speed of semantic analysis of literary vocabulary. In this paper, the RPA Adam model is proposed, which integrates the neighbor information in a convolution network to form a node representation vector, which contains not only the characteristics of its own nodes but also the characteristics of neighbor nodes. It is applied to graph convolution network of isomorphic network analysis, analyzes the types of devices that can be carried by embedded chips, and displays them with graphics.

\section{Semantic Analysis and Hard Decoding}

2.1. Overview of Word Cloud Semantic Analysis. Although word cloud semantic analysis data has good user and spatiotemporal characteristics, it also has some problems, such as sparse process records, low spatial accuracy based on word root location, and lack of clear semantic information [14]. If we can combine high-precision semantic data, or semantic microblog, Twitter social network data, and other data sources, the data complement each other; we can obtain more three-dimensional vocabulary perception [15]. The fusion, correlation, and error correction technology of largescale multispatiotemporal data will be a research difficulty. At present, most of the visual analysis based on mobile communication data is based on offline statistics or aggregation processing, while high timeliness services such as vocabulary disaster recovery need faster data processing capability $[16,17]$.

Hadoop distributed architecture (distributed model assistance technology in the distributed model collaboration framework, where each edge server does not need to publish its local data to other servers but can only use the intermediate parameters of model training), parallel processing algorithms, or optimization means of visual interaction. However, the total amount of research in this field is relatively small, and the improvement of analysis efficiency is an embedded research direction. Due to the complexity of vocabulary analysis goals and communication data, the threshold for users of vocabulary visualization analysis is relatively high. How to precipitate the existing research results, combined with artificial intelligence and domain knowledge, to design a more automatic visual analysis system for vocabulary analysis also has strong application value and significance [18]. In the era of big data, all kinds of network big data are in full bloom, with a large amount of information, strong accessibility, and wide communication power, which has become an irreplaceable advantage of network big data $[19,20]$. The feature semantic analysis model is used to improve the feature extraction structure of faster $r-c n n$, which improves the detection accuracy of the algorithm in remote sensing image ship [21]. At present, there is little research on feature extraction in water surface image target detection, which does not consider the geometric transformation of the target in different scenes and uses global information to enhance the feature semantics [22].

2.2. Lexical Root Convolution. Due to the unknown geometric transformation of the same object in different scenes or shooting angles, it will affect the feature extraction of the target detection algorithm. Therefore, the following optimization is proposed:

$$
\begin{gathered}
X A_{j}=\sum_{j=1}^{N} X_{i j}, \\
P_{i j}=\frac{X_{i j}}{X_{j}} .
\end{gathered}
$$

The variable convolution network structure diagram shows that the geometric shape of the water surface image is varied, and the convolution sampling points are shifted adaptively through the variable convolution. In order to solve the problem of bilinear interpolation, the global semantic information of a single feature graph is used to adaptively reorganize the upsampling features and break the constraint $P$ of the rule window:

$$
J U \sum_{i, j}^{N} f\left(X_{i j}\right)\left(v_{i}^{T} v_{j}+b_{i}+b_{j}-\log \left(X_{i j}\right)\right)^{2} .
$$

Traditional convolution $Q, T$ is divided into two steps:

$$
\begin{aligned}
& Q_{t 1}=\left(w_{1}^{1}, w_{2}^{1}, w_{3}^{1}, w_{4}^{1}, w_{5}^{1}\right), \\
& T_{t 2}=\left(w_{1}^{2}, w_{2}^{2}, w_{3}^{2}, w_{4}^{2}, w_{5}^{2}\right) .
\end{aligned}
$$

Backbone networks can enhance the adaptability to the geometric transformation of objects so as to extract more 
effective features from complex and changeable water surface images. The number of layers of the network is deep, the number of feature maps extracted from each layer is large, and the scale and semantic information are different. Therefore, it is necessary to carry out effective feature reorganization on the feature maps extracted from each layer of the backbone network, and it is of certain significance to use the global semantic information of the image [23]. Suppose the convolution kernel size is as follows:

$$
\cos (a, b)=\frac{a \cdot b}{|a| *|b|} .
$$

It facilitates the subsequent algorithm to detect the target through features [24]. Backbone network generally has dozens of convolution layers, and the output feature map of each layer has a certain multiscale detection ability and hierarchical structure [25]. The strategy of the feature semantic analysis model takes advantage of the characteristics of the backbone network and takes the feature maps of different scales and semantic strength output from some layers of the backbone network as the input to build a topdown multilayer feature map structure with the lateral connection. Therefore, the rule window used in the target detection of water surface image $A$ (Lang) can be defined as follows:

$$
\begin{aligned}
k_{t 1}[i] & =\sum_{j} \cos \left(w_{i}^{1}, w_{j}^{2}\right), \\
A(\text { Lang }) & =\frac{\mathrm{TP}+\mathrm{TN}}{\mathrm{TP}+\mathrm{TN}+\mathrm{FP}+\mathrm{FN}} .
\end{aligned}
$$

At present, this strategy has been adopted by many target detection networks, which improves the multiscale detection ability of the detection algorithm and improves the accuracy obviously. In the top-down structure of the feature semantic analysis model, bilinear interpolation is generally used for upsampling. Bilinear interpolation upsampling only determines the upsampling core by the spatial position of the pixel. The receptive field is very small, and it does not use global semantic information, so it is a uniform upsampling method [26]. In the era of traditional target detection, many scholars rely on the sea antenna and another image global information for the detection of water surface targets:

$$
\begin{aligned}
L_{t 1} & =\mathrm{AD} * \operatorname{Test}\left(k_{t 1}\right), \\
A D & =\frac{\mathrm{TP}}{\mathrm{TP}+\mathrm{FP}}
\end{aligned}
$$

where 1 is the enumeration of the positions listed in AD. In variable convolution, the position offset listed in TP is given as follows:

$$
\begin{aligned}
\eta N \lambda(A i, A j) & =\left[\log \left(\frac{\left|x_{A_{i}}-a_{A_{j}}\right|}{w_{A_{j}}}\right), \log \left(\frac{\left|y_{A_{i}}-y_{A_{j}}\right|}{h_{A_{j}}}\right), \log \left(\frac{w_{A_{i}}}{w_{A_{j}}}\right), \log \left(\frac{h_{A_{i}}}{h_{A_{j}}}\right)\right], \\
\varpi \mathrm{WN} & =\frac{\mathrm{TP}}{\mathrm{TP}+\mathrm{FN}} .
\end{aligned}
$$

Among $\eta N \lambda(A i, A j)$ the amplitude coefficient wn of each sampling position is given as follows:

$$
\begin{aligned}
L_{i} & =\operatorname{attention}\left(u_{i}\right), \\
E_{1}^{i j} & =\cos \left(S_{1}^{i}, S_{2}^{j}\right) .
\end{aligned}
$$

Because 1 may be a small amount, the position coordinates of the offset sampling points are not an integer, so bilinear interpolation is necessary:

$$
c_{i j}=\frac{e^{b_{i j}}}{\sum_{k} e^{b_{i k}}},
$$

where

$$
u_{(j \mid i)}=w_{i j} A_{i} .
$$

Enumerate all the position coordinates on the input characteristic graph $X ; G$ is a bilinear interpolation kernel function, which satisfies the following:

$$
s_{j}=\sum_{i} c_{i} j u_{(j \mid i)} .
$$

In order to obtain CNN adaptively, a layer of a traditional convolutional neural network is paralleled in the convolution process:

$$
\begin{aligned}
\operatorname{CNN}(k) & =\frac{\|k\|^{2}}{1+\|k\|^{2}} \frac{k}{\|k\|^{\prime}} \\
h_{t} & =\tanh \left(w_{c} x_{t}+u_{c}\left(r_{t} \Theta h_{t-1}\right)+b_{c}\right), \\
h_{t} & =z_{t} \Theta h_{t-1}+\left(1-z_{t}\right) \Theta h_{t} .
\end{aligned}
$$

The HT is the convolution kernel of $3 \mathrm{D}$ migration, and the position amplitude coefficient, lateral offset, and longitudinal offset are obtained by decomposition. Through parallel convolution layers, variable convolution can adaptively obtain the offset parameters of convolution sampling points. Feature reorganization of semantic information is divided into two modules: adaptive generation module of upsampling kernel and feature reorganization module [27]. In the upsampling kernel adaptive generation module, first of all, in order to reduce the amount of calculation in the 
subsequent operation, the input characteristic graph with width and height channel HWC is compressed:

$$
v_{j}=\operatorname{squash}\left(s_{j}\right) \text {. }
$$

Then, by convolution, the kernel size $B$ is as follows:

$$
b_{i j}=b_{i j}+u_{(j \mid i)} v_{j}
$$

The size of the convolution layer is as follows:

$$
\sigma_{\mathrm{ikjl}}= \begin{cases}\frac{n}{\Delta_{\mathrm{ikjl}}} \sqrt{\sum_{s=1}^{n}\left(x_{\mathrm{ik}}(\varepsilon)-x_{\mathrm{jl}}(\varepsilon)\right)^{2} \Delta_{\mathrm{ikjl}}(\varepsilon),} & \Delta_{\mathrm{ikjl}}>0 ; \\ 0, & \Delta_{\mathrm{ikjl}}<0 .\end{cases}
$$

The number of upsampling convolution kernels is as follows:

$$
\Delta_{\mathrm{ikjl}}=\sum_{\delta=1}^{n} \Delta_{\mathrm{ikjl}}(\varepsilon) .
$$

Finally, all upsampling kernels are normalized so that the sum of their weights is 1 . The feature recombining module receives the upsampling kernel output from the upsampling kernel adaptive generation module and maps each upsampling kernel from the input feature graph:

$$
z_{t}=\sigma\left(w_{z} x_{t}+u_{z} h_{t-1}+b_{z}\right)
$$

The results show that the width and height channels are 1

$$
r_{t}=\sigma\left(w_{r} x_{t}+u_{r} h_{t-1}+b_{r}\right)
$$

Because the sampling core is generated adaptively based on the global semantic information, in the process of upsampling, the features in the feature map can be reorganized by using the self-adaptive acquired semantic information so as to output the feature map with strong semantic information. Through the feature reorganization based on semantic information, the obtained feature map is fused under the global receptive field so that the target image can be extracted. The target feature has a global field of view and information so as to improve the target detection accuracy of water surface images.

\subsection{Hardware Decoding Semantic Feature Extraction} Structure. Through variable convolution and feature reorganization based on semantic information, this paper proposes a hardware decoding semantic feature extraction structure. In variable convolution and feature reorganization of semantic information for input images, the first step is to extract four feature images of different scales through one layer of residual block and three layers of variable convolution residual block. The residual block is composed of traditional convolutional identity mapping, and the variable convolutional residual block is composed of variable convolutional identity mapping; then, the feature map of the previous scale is recombined according to the semantic information and then the channel is connected with the next one. Scale feature maps are superimposed; finally, four layers of feature maps with strong semantic information are obtained to form a feature semantic analysis model for subsequent target detection tasks [28]. Hardware decoding uses node or edge type to determine the weight matrix. For those relationships that do not appear enough times, it is difficult to learn the accurate relationship-specific weight, which leads to the model cannot fully mine the semantics of the interaction between relationships in the heterogeneous graph. HGT proposes a method based on message passing, in which high-order adjacency information is included through a multilayer graph convolution network. In order to solve the problem of heterogeneity, an attention mechanism is introduced by depending on the types of nodes and edges. Moreover, combined with relative time coding, it can deal with dynamic heterogeneous graphs and enhance HGT [29]. In order to deal with large-scale data, HGT designs a heterogeneous subgraph sampling algorithm hg sampling. This algorithm is different from the traditional sampling algorithm, which can make the sampling of different node types keep similar distribution, and the information loss is small. The method of adaptive heterogeneous information does not need domain experts to define the path of the element and directly carries out modeling and analysis on the heterogeneous graph. Among them, hetgnn samples neighbors by random walk and aggregates them by neighbor type, but its computational complexity is high. To solve this problem, hetsann transforms different types of nodes into the same semantic space, aggregates nodes through attention mechanism, and analyzes heterogeneous networks directly. Compared with the model of the sensing node type, rshn is a convolutional network of heterogeneous graphs sensing the relationship structure, which can obtain the hidden information between the adjacent nodes in the heterogeneous graph and enhance the embeddedness of the network. Activehne decomposes the heterogeneous network into several subgraphs with only two kinds of nodes for modeling and analysis and does not need a random walk sampling sequence. For heterogeneous networks with multiple relationships and types of nodes, $\mathrm{r}$-gen and compgen allocate learnable matrices for a certain type of nodes or edges, but they cannot fully mine heterogeneous information. HGT adopts the attention mechanism of a node type and edge type dependence. Nodes connected by different edge types can transfer and interact information and can obtain high-order information across different levels. HGT can also deal with dynamic heterogeneous networks with relative time coding. However, most adaptive heterogeneous information networks have many parameters, resulting in high computational complexity and difficult training. Some scholars have tried to solve this problem. For example, compgen uses decomposition operation to express the relationship as a group of weighted combinations so that the number of parameters is only related to the number of bases.

\section{Semantic Recognition of Literary Words}

3.1. Research Content. In this paper, based on the embedded microsystem, combined with the design idea of robot process automation (RPA), a CNN logic algorithm is added to improve 
the accuracy and speed of semantic analysis of literary vocabulary. In this paper, the RPA Adam model is proposed, which integrates the neighbor information of the convolutional network to form a node representation vector, which contains not only the characteristics of its own nodes but also the characteristics of neighbor nodes. It is applied to graph convolutional networks with isomorphic network analysis. The aggregation methods include equalization, maximization, LSTM, and attention mechanism aggregation.

3.2. Experimental Design. Embedded systems are described as application-centric. Software and hardware can be cut to adapt to the application system's comprehensive and strict requirements for function, reliability, cost, volume, power consumption, and other special computer systems. This paper starts from the embedded language analysis network of literary vocabulary, first introduces the relationship between semantic analysis of literary vocabulary and language analysis network of literary vocabulary, and through the analysis of the connotation of communication, expounds that semantic analysis of literary vocabulary may become a new paradigm of embedded communication technology. Then, it introduces the basic model and composition of semantic analysis of literary vocabulary.

The flow chart of RPA Adam model is shown in Figure 1. Compared with the benchmark structure, the feature extraction structure increases the number of parameters by about $7 \mathrm{~m}$; the floating-point computation increases by about 10 gflops; compared with each detection algorithm, the time complexity increases by about $2 \%$; and the frame rate decreases by about $10 \%$. Therefore, the improved structure has little impact on the real-time performance of the detection algorithm. Fast RCNN and cascade RCNN are widely used twostage target detection algorithms, and retinanet is a widely used one-stage target detection algorithm.

By analyzing the limitations of point-to-point semantic analysis of literary vocabulary, this paper holds that the semantic analysis network of literary vocabulary based on knowledge sharing and resource integration is more suitable to be the basis of the literary vocabulary language analysis network. Furthermore, this paper analyzes the basic components of the semantic analysis network of literary words and introduces a networking example of a semantic analysis network of literary words based on federal edge intelligence. The simulation results show that the literature vocabulary semantic analysis network is expected to ensure data security, and at the same time, greatly reduces resource utilization and improves communication efficiency. Finally, it discusses the openness of the embedded development of the semantic analysis network of literary vocabulary. In use, people can enjoy ubiquitous computing, storage, and communication services without carrying exclusive computing and communication devices such as mobile phones or computers.

\section{Results and Discussion}

4.1. Semantic Analysis of Literary Words. As there are certain differences between Chinese and English, the conversion and analysis order between the two is English and then
Chinese. For semantic analysis, the first is the analysis of English words. Since the encoding of Chinese characters is different from that of English, it is necessary to segment words first and analyze the semantics in combination with the meaning of the words. Therefore, this article first introduces common English semantic analysis and then explains the Chinese semantic analysis model.

As shown in Figure 2, the semantic first-word segmentation is expected to greatly improve the communication efficiency and the quality of user experience (QoE) by integrating the user's information needs and semantics into the communication process, Quality of experience), and fundamentally solve the problems of cross system, cross protocol, cross network, cross man-machine incompatibility, and difficult intercommunication in traditional data based communication protocols, so as to truly realize the grand vision of "all things transparent literary language analysis," that is, the seamless integration of communication network, computing, storage, and other software/hardware devices into life.

As shown in Figure 3, compared with the traditional digital signals mainly composed of 0 and 1 , semantic signals may contain more information and express more content. The content that users in different regions and time may convey and understand will also be affected by various complex factors, such as the personality and emotion of the disseminator and the receiver, the communication environment, the history of interaction with the surrounding users and environment, and the semantic context. On the other hand, the context in which semantic analysis of literary words takes place and the social and communication history of users can effectively help users to better identify semantics and reduce semantic noise.

As shown in Figure 4, in the classroom, the semantic information communicated between teachers and students is most likely limited to classroom knowledge, while in the road or intersection, the semantic information communicated between driverless vehicles and driverless vehicles is most likely focused on vehicle driving behavior and traffic conditions. Therefore, only considering the content of pointto-point semantic analysis of literary words and ignoring the influence of surrounding users and communication network environment will greatly limit the efficiency of knowledge recognition and processing in semantic analysis of literary words.

As shown in Figure 5, the current mainstream work of semantic analysis of literary vocabulary usually emphasizes the use of prior knowledge to reduce communication costs and improve the success rate of semantic transmission but ignores the cost of computing and storage resources needed to identify, extract, and interpret semantic information. The latest report shows that the resources consumed by the most advanced artificial intelligence algorithms have been increasing rapidly in the past few years. The cost will double every few months, and the resource cost of advanced artificial intelligence algorithms will increase by 200000 times. The amount of computing and storage resources required by these artificial intelligence algorithms is far beyond the computing and storage capacity of current mainstream 


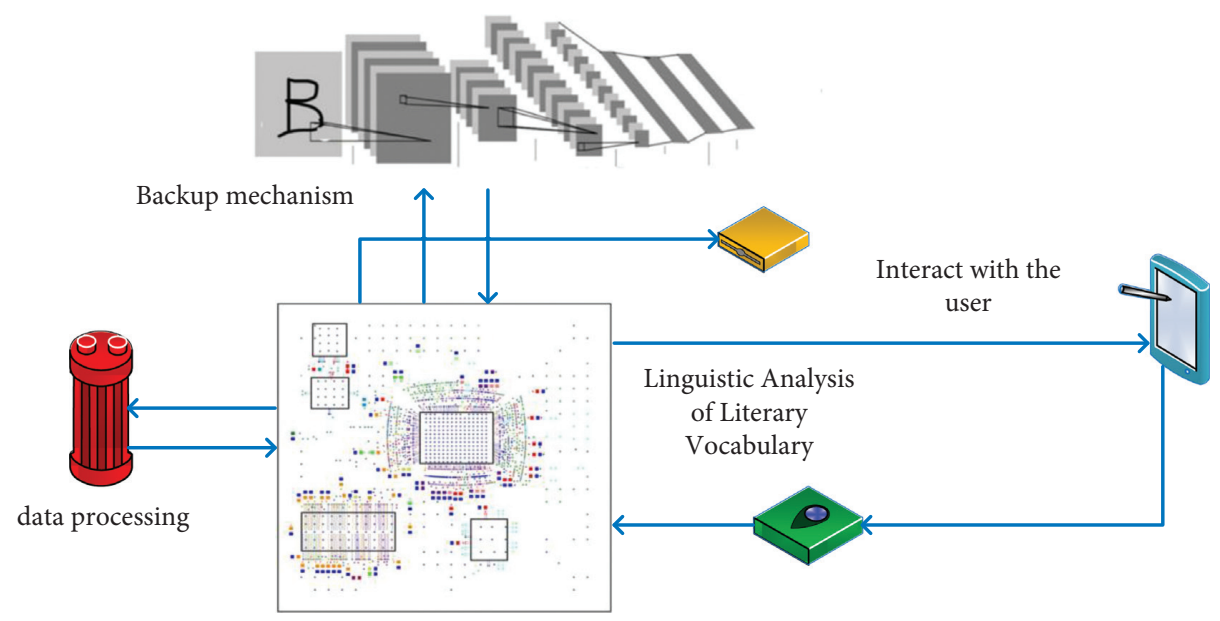

FIgURE 1: RPA-Adam model (some pictures are quoted from CNKI, cnki.net).

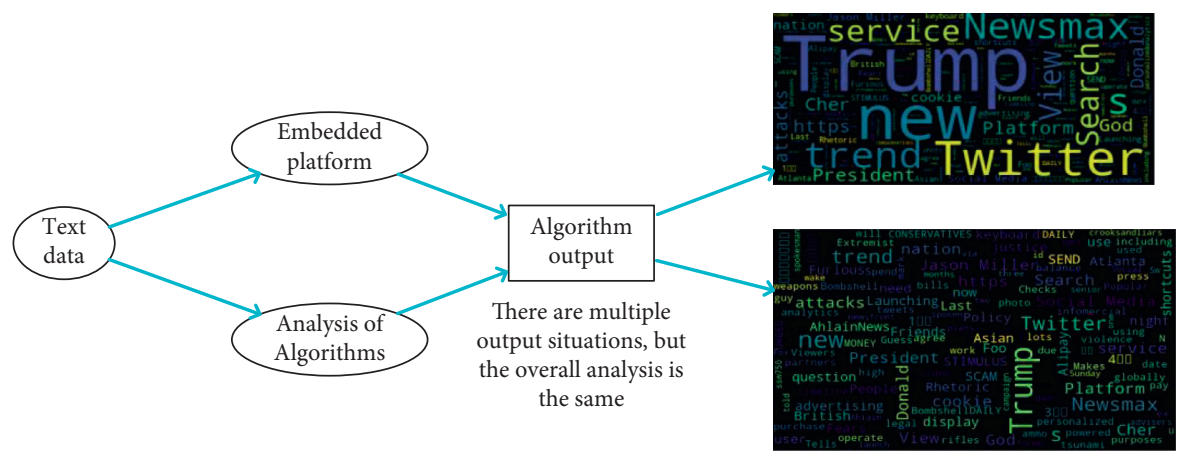

FIGURE 2: Semantic word segmentation process.

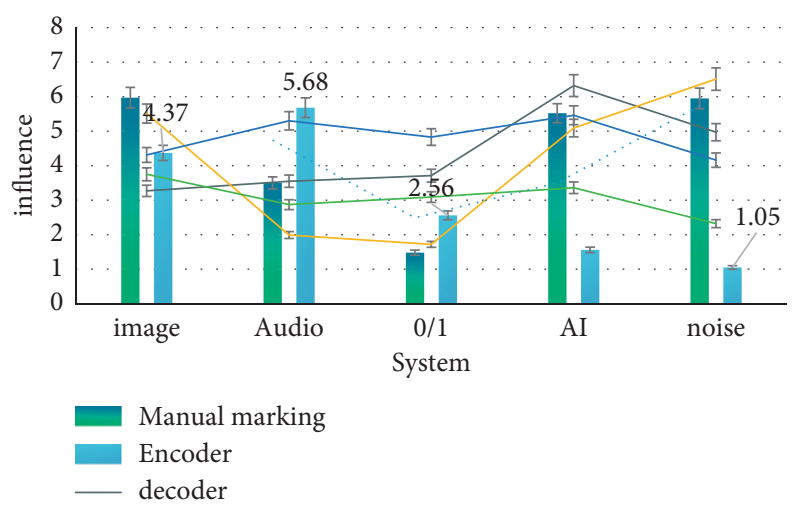

Figure 3: Context is difficult to perceive and difficult to recognize.

terminal devices, which further restricts the application scenarios of point-to-point literary vocabulary semantic analysis.

A high-performance GPU is used to train three current mainstream image data sets and a simple convolution neural network. The accuracy and time-consuming of the training model are shown in Table 1.

As shown in Figure 6, in order to reduce the cost of deploying computing and storage resources for a single user, in this structure, each user can unload tasks that consume a lot of resources, such as semantic coding and decoding, to its nearest edge server. It can be trained by two or more semantic models, one is a global learning model and the other is a local learning model. In this paper, a knowledge map is used as the representation method of semantics. The semantic analysis network architecture of literature vocabulary mainly includes the following elements: massive literature vocabulary semantic analysis participants (users), edge computing server, and global knowledge and semantic recognition model collaborator. The edge server is a high- 


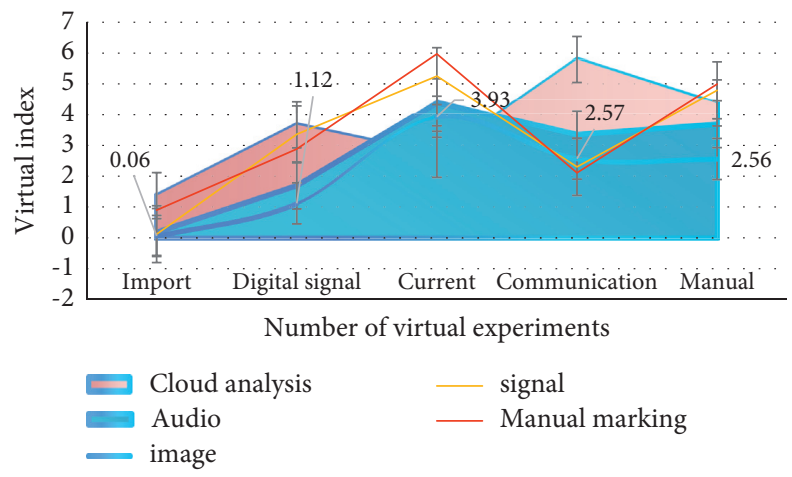

FIgURE 4: Communicated semantic information.

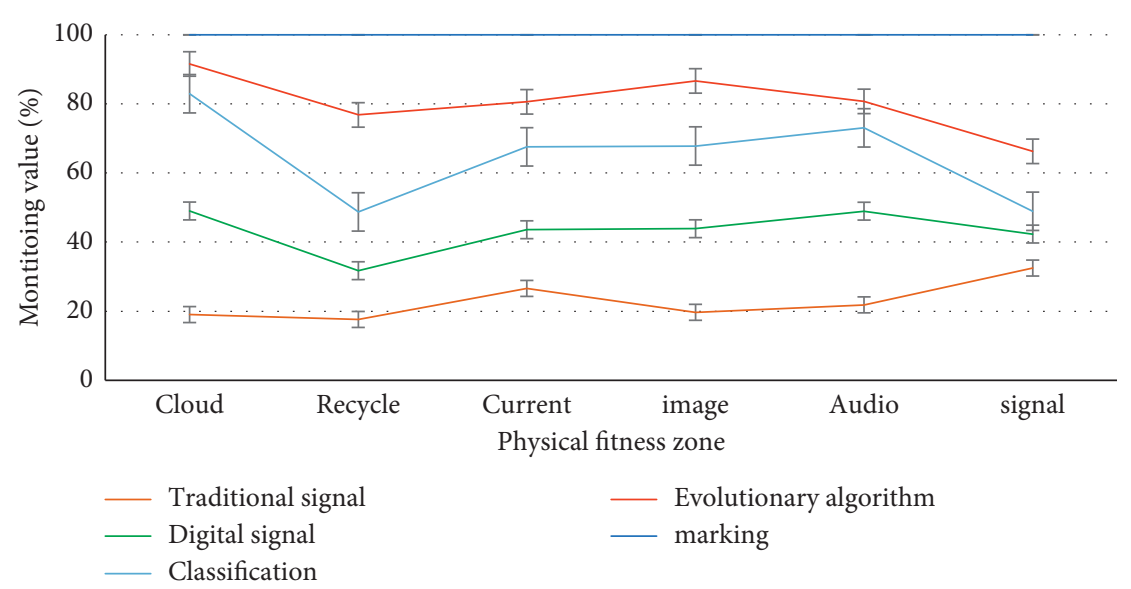

FIGURE 5: The key to the work of semantic communication.

TABle 1: Convolutional neural network training model accuracy and time-consuming.

\begin{tabular}{lccccc}
\hline Item & Traditional signal & Digital signal & Classification & Accuracy & Times \\
\hline GPU & 1.28 & 0.12 & 0.23 & 0.04 \\
Network & 1.72 & 2.83 & 3.24 & 2.14 & 2.79 \\
Composition & 4.36 & 2.55 & 4.01 & 4.58 \\
Image & 1.77 & 3.8 & 2.24 & 4.37 \\
Audio & 2.74 & 4.13 & 4.02 & 3.59 \\
\hline
\end{tabular}

performance computing server deployed near users, which can perform computing, storage, AI model training, and other tasks.

As shown in Figure 7, unlike information theory which mainly uses discrete digital signals of 0 and 1 to describe information, for knowledge map, when the data uploaded by users cannot be accurately recognized by all local models, train a new semantic recognition model. The information or event expressed by the user may be a subgraph existing in the knowledge graph. Therefore, each possible subgraph of the knowledge graph can be regarded as a random variable, and all possible subgraph sets can be regarded as a symbol set. When each edge computing server receives a user's request, it first searches for locally trained knowledge entities and relationship recognition models. In order to further improve the search speed of the semantic knowledge model, each user should perceive the surrounding environment, communication time, specific scene, and other information related to the meaning of communication before performing the encoding and decoding process. The information will be uploaded to the edge computing server together with the source signal to analyze the specific scene of literary vocabulary semantic analysis so as to reduce the number of semantic encoders and decoders in the search space of the model. In order to coordinate knowledge modeling among multiple edge servers and protect local semantic data from leakage, this paper adopts a distributed model collaboration framework based on federated learning, in which each edge server does not need to publish its local data to other servers but can only use the intermediate parameters of model training, such as gradient or AI. In this way, data privacy and training quality can be guaranteed, and the storage and 

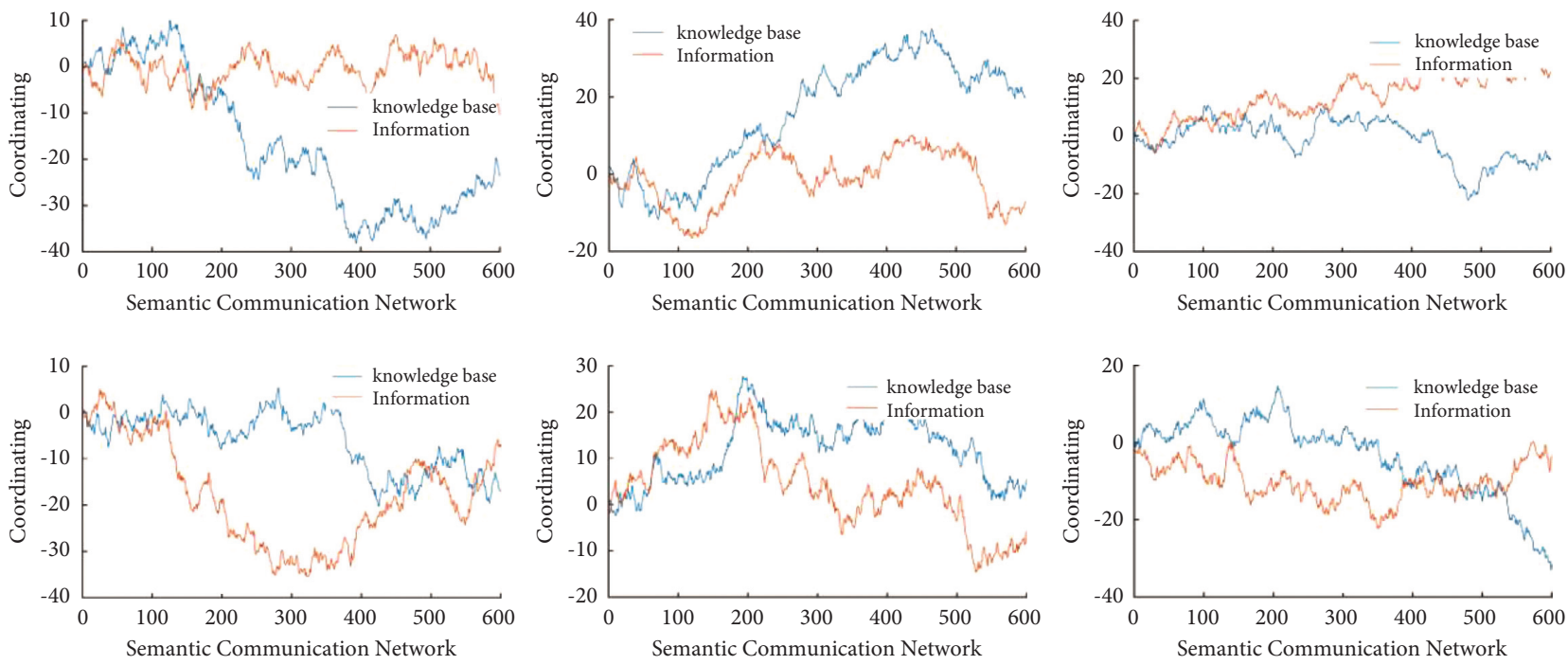

FIgURE 6: Contradictory semantics between privacy and security.

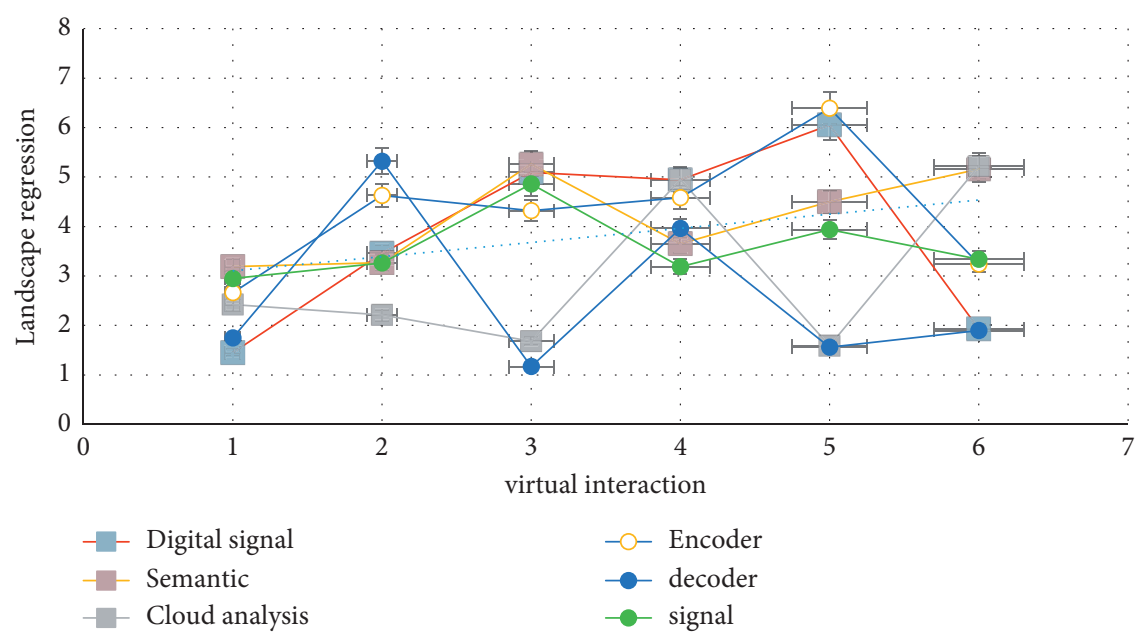

FIgURE 7: Information or events expressed by users.

computing load of a single edge server can be further reduced. Specifically, when the entity recognition model of federal intelligent edge training is adopted, the storage and computing time required by a single edge computing server are shown in Table 2.

As shown in Figure 8, using multiple edge computing servers can effectively reduce the time required for model training and the amount of labeled data that each edge computing server needs to store. Another advantage of using a knowledge map to represent a semantic knowledge base is that the semantic information can be compressed by using the correlation between knowledge entities. Assuming that the transmitter only transmits part of the knowledge entity class information, the receiver can recover all the knowledge entity class information by using a semisupervised graph convolution neural network model after receiving part of the knowledge entity label data set sent by the transmitter. All data sets are processed by a network structure with two layers. Each layer obtains a new feature vector by aggregating the feature vectors of the target node and neighbor nodes as the output, and the receiver can use the final feature vector for category prediction. The complete adjacency matrix and the node feature vector of the graph are stored in the form of a sparse matrix, so the amount of data occupied is very small.

As shown in Figure 9, the RPA Adam model is used for training. The number of training iterations is 600 , and the training stops when the loss does not decrease in 10 consecutive iterations. The number of hidden units transmitted between the two layers of the network is 16 . The recovery error probability of the knowledge map recovered by the receiver at different data compression rates is analyzed. It can be observed that the error rate is the same under different compression rates because of the different correlations among knowledge entities in different data sets. Specifically, high frequency can maintain a low error rate of $10.79 \%$ when the compression rate is $4.85 \%$, but when the compression 
TABLE 2: The amount of storage required for edge computing servers.

\begin{tabular}{lccccc}
\hline Item & Image & Audio & Model & AI & Noise \\
\hline Manual marking & 5.2 & 3.58 & 2.44 & 4.85 & 2.74 \\
Encoder & 5.38 & 2.43 & 4.84 & 4.14 & 1.62 \\
Decoder & 1.21 & 1.1 & 4.83 & 6.81 & 2.83 \\
Cloud analysis & 5.44 & 2.37 & 3.53 & 3.44 \\
Digital signal & 2.7 & 5.58 & 4.28 & 3.19 \\
Design & 2.03 & 1.53 & 4.73 & 3.53 & \\
\hline
\end{tabular}

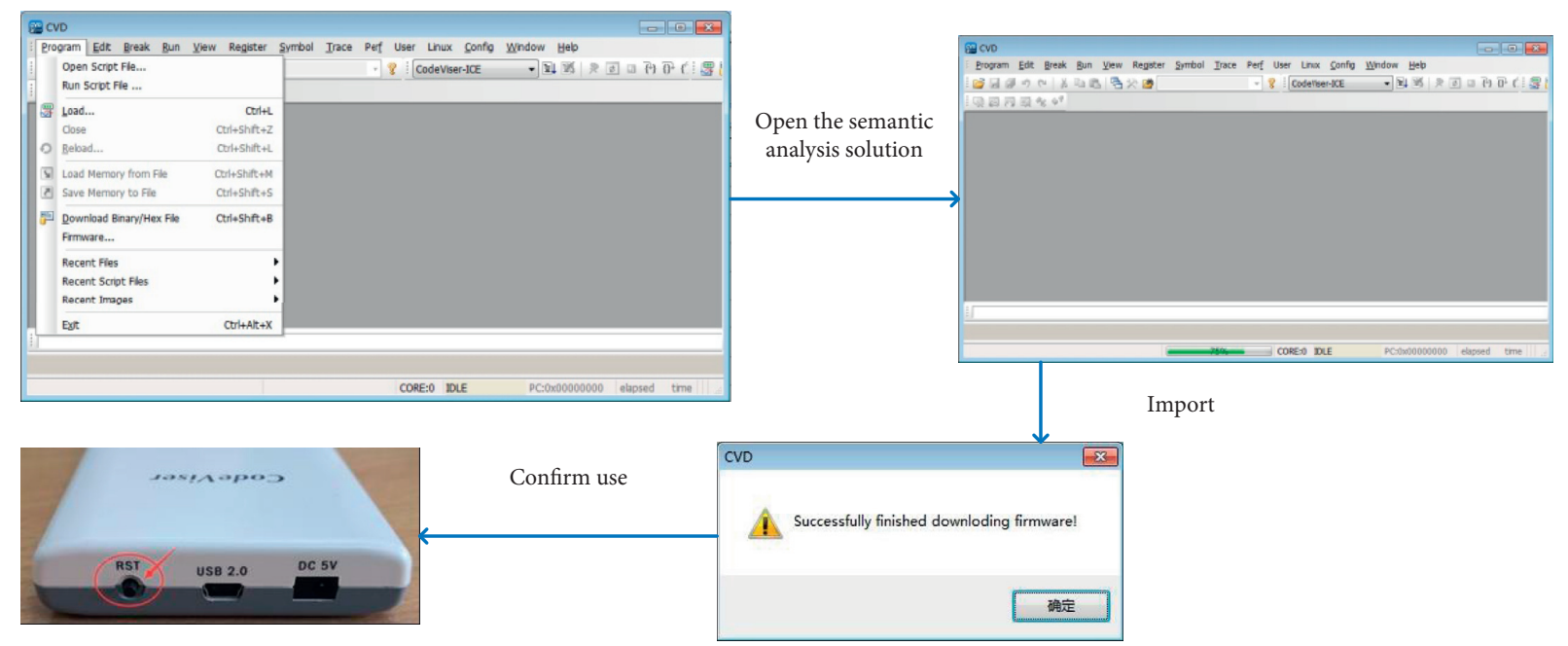

Figure 8: Embedded chips are used in various embedded devices (some pictures quoted from Daguan data, datagrand.com).

rate of high frequency is only $60.32 \%$, the error rate is as high as $11.26 \%$, while the compression rate of low frequency is $23.51 \%$ when the error rate is $9.65 \%$.

The traditional text and voice interaction cannot meet the demand, and a large number of emerging $\mathrm{H} 2 \mathrm{H}$ literature language analysis technologies such as $3 \mathrm{D}$ holographic projection and tactile Internet will gradually become popular and occupy an increasingly important position in the embedded communication network. As shown in Figure 10, with the emergence, promotion, and popularization of a large number of new services, the existing network architecture will be difficult to meet the rapid growth of the diverse needs of resource service users under different literary language analysis methods. Although the abovementioned literary language analysis interface and technology can greatly improve the user experience and greatly expand the business scope and application scenarios of the communication network, there is still a lack of a standardized communication network architecture that can unify the above three literary language analysis methods.

Therefore, it is possible to reduce the frequency of semantic transformation and semantic analysis between literature and $\mathrm{H} 211$, as shown in the semantic analysis of $\mathrm{H} 211$ (Figure 11). It can be described by the following mathematical model. $X$ is defined as a finite set of codewords that may be transmitted, and $X$ is used to represent a discrete random variable (such as the digital sequence of 0 and 1) of codewords that may be transmitted. Similarly, $s$ is defined as the finite set of semantic information that may be sent, and $S$ is used to represent the discrete random variables that may have semantic information in the source signal.

4.2. Discussion. The semantic encoder detects and extracts the specific semantic content contained in the source signal and compresses and removes the semantic independent information. The encoder should also have the ability to detect the knowledge differences between the source end and the terminal end and may infer the knowledge entities and associations shared by both sides of the communication through logic or other methods. For example, when an adult communicates with a child or an adult, he or she usually uses different vocabularies and expressions to ensure that the users participating in the communication share the same knowledge base. The encoder should also be able to process different types of source signals. For example, when the source signal is a picture or audio, the encoder should first recognize the entities in the source image and audio according to the local knowledge owned by the signal source and the signal receiver and then recognize the possible relationship between the entities through a common or similar knowledge model; the accuracy of entity and relation recognition directly affects the performance of semantic analysis of literature words between source and sink. In other words, it is different from the traditional communication theory, which only considers technical problems. In 

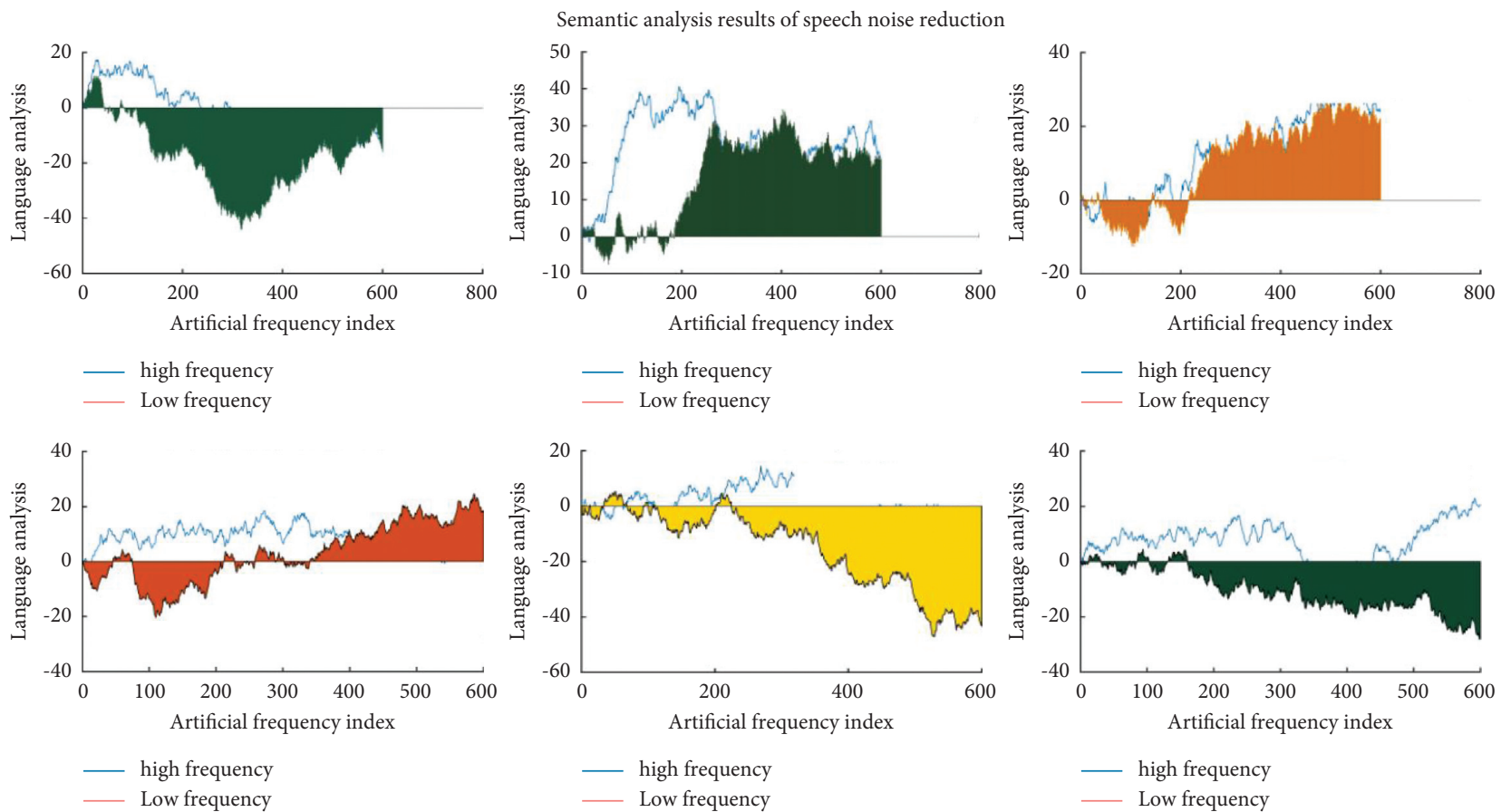

Figure 9: Semantic analysis results of speech noise reduction.

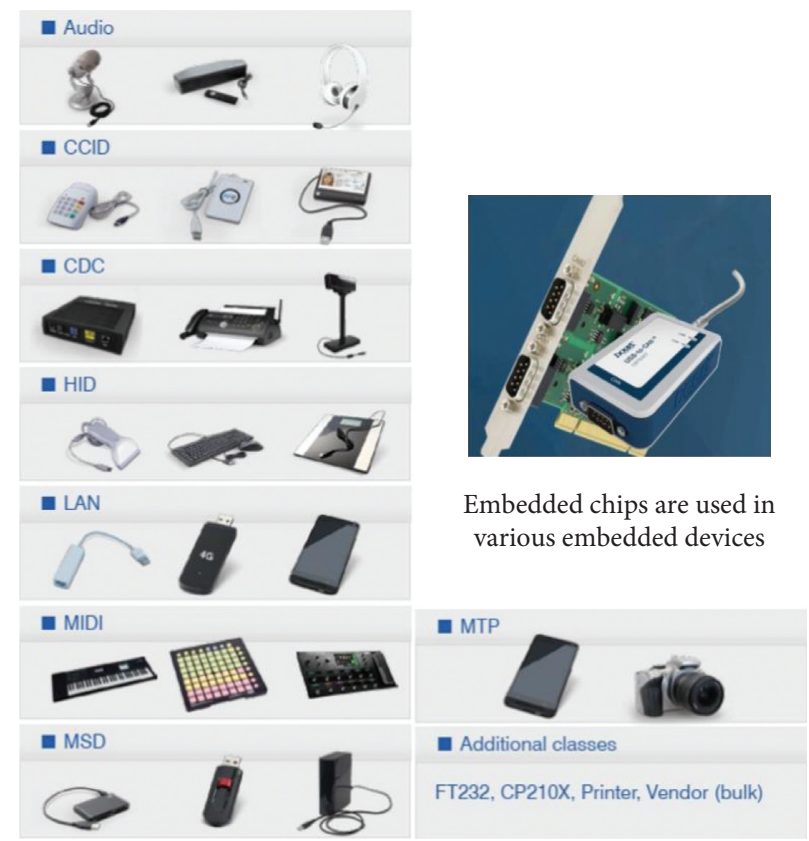

FIGURE 10: Chip and its application products (some pictures quoted from Daguan data, datagrand.com).

the semantic analysis of literary words, communication performance is no longer mainly measured by the number of resources such as frequency spectrum, energy consumption, and the signal-to-noise ratio of the received signal but needs to comprehensively consider the impact of various factors such as the amount of computation, storage, model accuracy, and label data set size on semantic recognition, transmission, and recovery. These are difficult to characterize and analyze by simply extending the traditional Shannon formula.

The semantic decoder interprets the information sent by the source and restores the received signal to a form that the user can understand. The decoder also needs to evaluate the user satisfaction of the receiving end so as to judge whether the received semantic information is correct. The decoders can also feed back the relevant information (such as the 


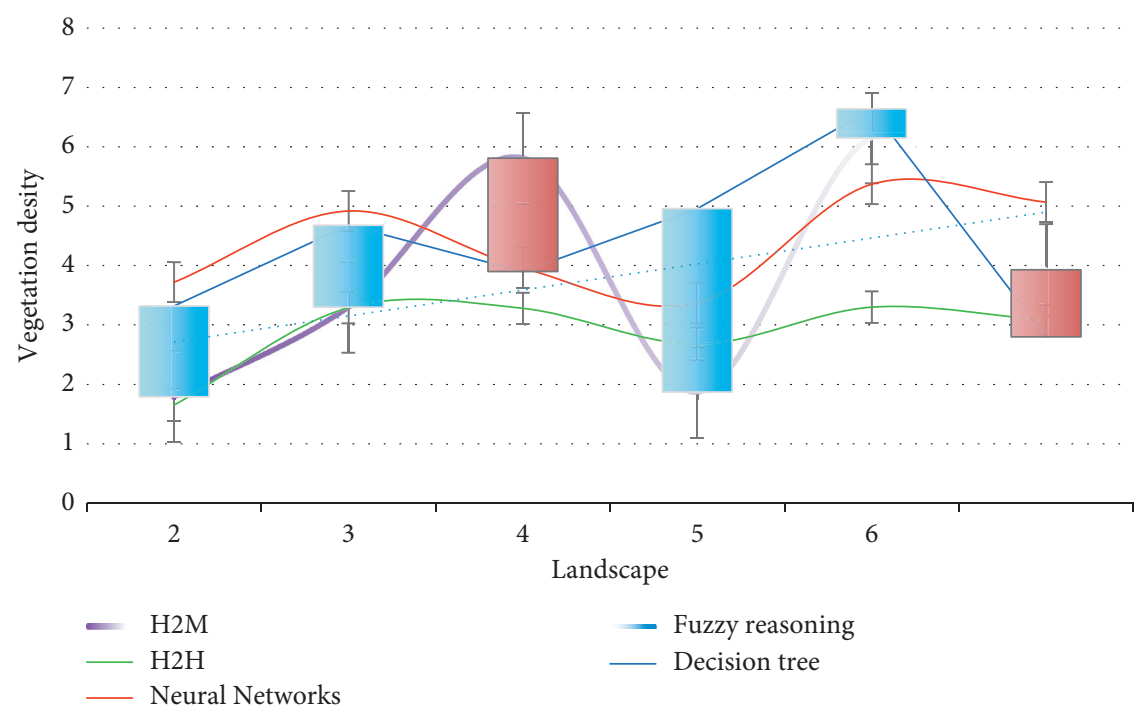

Figure 11: Human universal knowledge and semantic system.

evaluation score) to the encoder to further improve the entity and relationship recognition model in embedded communication. Semantic noise is a kind of noise introduced in the process of semantic analysis of literary words, which may lead to incorrect recognition and interpretation of semantic information. It can be generated in the process of coding, transmission, and decoding. In the process of coding, semantic noise may be caused by the wrong recognition of the entities in the source signal and the relationship between them. Channel fading and noise may also cause data loss and semantic distortion. In the process of decoding, the wrong interpretation of semantic information and the misunderstanding of users may also lead to semantic noise. Although semantic analysis of literary vocabulary has great potential, its development still faces the following challenges.

The first prerequisite for semantic analysis of literary vocabulary is that all communication participants (transmitter and receiver) can share one or more universal semantic knowledge bases, which generally include three levels of knowledge base sharing. First of all, the transmitter and receiver need to have a consistent knowledge entity and relationship base. Secondly, when the knowledge base of transmitter and receiver is not completely consistent, the communication content of transmitter and receiver should be within the common knowledge scope of both sides. In other words, the transmitter and receiver should have the ability of knowledge and background cooperation and difference recognition. Finally, when the transmitter and receiver detect unknown semantic knowledge entities and relationships, they need to have the ability of knowledge base collaborative update. It is impossible for a single user to update and maintain a unique knowledge base independently, but all communication participants need to maintain and update the relevant information of semantic knowledge. For example, even for human beings with strong information processing and memory ability, knowledge accumulation and recognition ability also need to spend decades of learning, exploration, and practice. For electronic equipment and machines with only a few years or even shorter service life, each equipment needs to maintain and update the knowledge base separately, which not only costs huge communication and storage costs but also costs a lot of money, and knowledge accumulation and data acquisition also need a lot of time. These factors make it difficult to promote the semantic analysis of literary vocabulary.

\section{Conclusions}

Semantic analysis cloud computing (dozens of natural language core algorithms and solutions, comprehensive coverage of various needs for language processing, standardized interface packaging, rapid use of tools through cloud computing, greatly reducing development labor costs). For the first time, a new service-based architecture (SBA) was introduced to meet the different needs of massive vertical businesses. It aims to realize the transformation of an embedded microsystem communication network from a traditional architecture that only pursues high transmission rates to a new architecture that can support three business scenarios and multiple vertical industries. It is augmented reality/virtual reality technology, large-scale Internet of things and Internet of things-semantic analysis and other emerging application scenarios to provide protection for the promotion and popularization. In the embedded era, when all physical layer dimensional resources are nearly saturated, how to further improve the communication efficiency and continuously meet the needs of complex, diverse, and intelligent information transmission is a new challenge for the development of wireless technology. In recent years, these emerging services no longer only rely on high-speed data transmission but gradually put forward higher requirements for network intelligence and service diversity. With the rapid growth of people's demand for smart wireless communications, various emerging smart services based on wireless communications technologies emerge in an endless stream 
(such as industrial Internet, smart connected cars, telemedicine/surgery, virtual reality, and holographic projection technology, etc.). Driven by this development trend, embedded communication networks will gradually transform into a new architecture of literature vocabulary language analysis, which is highly automated, intelligent, and can provide more close to the needs and experience of human users.

\section{Data Availability}

The data that support the findings of this study are available from the corresponding author upon reasonable request.

\section{Conflicts of Interest}

The authors declare no potential conflicts of interest with respect to the research, authorship, and/or publication of this article.

\section{Acknowledgments}

The authors received no financial support for the research, authorship, and/or publication of this article.

\section{References}

[1] E. F. Codd, "A relational model of data for large shared data banks," Communications of the ACM, vol. 13, no. 6, pp. 377-387, 2020.

[2] I. Androutsopoulos, G. D. Ritchie, and P. Thanisch, "Natural language interfaces to databases-an introduction," Natural Language Engineering, vol. 1, no. 1, pp. 29-81, 2020.

[3] K. Affolter, K. Stockinger, and A. Bernstein, "A comparative survey of recent natural language interfaces for databases," The VLDB Journal, vol. 28, no. 5, pp. 793-819, 2019.

[4] L. Blunschi, C. Jossen, D. Kossmann et al., "SODA: generating SQL for business users," Proceedings of the VLDB Endowment, vol. 5, no. 10, pp. 932-943, 2020.

[5] G. Zenz, X. Zhou, E. Minack et al., "From keywords to semantic queries-incremental query construction on the semantic web," Journal of Web Semantics, vol. 7, no. 3, pp. 166-176, 2020.

[6] D. Saha, A. Floratou, K. Sankaranarayanan et al., "ATHENA: an ontology-driven system for natural language querying over relational data stores," Proceedings of the VLDB Endowment, vol. 9, no. 12, pp. 1209-1220, 2020.

[7] L. Fei and H. Jagadish, "Constructing an interactive natural language interface for relational databases," Proceedings of the VLDB Endowment, vol. 8, no. 1, pp. 73-84, 2020.

[8] C. Zhao and J. Li, "Equilibrium selection under the bayesbased strategy updating rules," Symmetry, vol. 12, no. 5, p. 739, 2020.

[9] Y. Wu, F.-X. Zhu, and J. Zhou, "Using probabilistic graphical model for text sentiment analysis," Journal of Chinese Computer Systems, vol. 36, no. 7, pp. 1421-1425, 2020.

[10] Z. Zhang, L. Lin-Chuan, Z. Xiang-Qi et al., "Aspect sentiment analysis combining ON-LSTM and self-attention mechanism," Journal of Chinese Computer Systems, vol. 41, no. 9, pp. 1839-1844, 2020.

[11] C. Hu, B.-Y Gao, C. Lian-Na et al., "Sentiment classification model combining attention mechanism and bidirectional slice
GRU," Journal of Chinese Computer Systems, vol. 41, no. 9, pp. 1793-1799, 2020.

[12] D. Bahdanau, K. Cho, and Y. Bengio, "Neural machine translation by jointly learning to align and translate," Computer Science, vol. 24, no. 5, pp. 1345-1349, 2020.

[13] X. Liu, Y. Li, and Q. Wang, "Multi-view hierarchical bidirectional recurrent neural network for depth video sequence based action recognition," International Journal of Pattern Recognition and Artificial Intelligence, vol. 32, no. 10, p. 1850033, 2018.

[14] G. D. Lorenzo, M. Sbodio, F. Calabrese et al., "AllAboard: visual exploration of cellphone mobility data to optimise public transport," IEEE Transactions on Visualization and Computer Graphics, vol. 22, no. 2, pp. 1036-1050, 2020.

[15] P. W. Zhang, E. Weinan, X. C. Yuan, and Y. Fu, "Big data analysis and application technology innovation platform," Big Data Research, vol. 4, no. 4, pp. 86-93, 2018.

[16] G. Sagl, M. Loidl, E. Beinat et al., "A visual analytics approach for extracting spatio-temporal urban mobility information from mobile network traffic," International Journal of GeoInformation, vol. 1, no. 3, pp. 256-271, 2020.

[17] N. Chen, B. Rong, X. Zhang, and M. Kadoch, "Scalable and flexible massive MIMO precoding for 5G H-CRAN," IEEE Wireless Communications, vol. 24, no. 1, pp. 46-52, 2017.

[18] W. B. Huang, S. C. Xu, L. Ma et al., "Analysis of mobile user behaviors with telecommunication data," New Technology of Library and Information Service, vol. 5, no. 5, pp. 80-87, 2020.

[19] Y. N. Li, C. F. Ao, Y. D. Wu et al., "Visual analysis of user behavior based on the mobile phone data," Journal of Southwest University of Science and Technology, vol. 32, no. 4, pp. 60-67, 2020.

[20] Z. Lv, R. Lou, J. Li, A. K. Singh, and H. Song, "Big data analytics for $6 \mathrm{G}$ enabled massive internet of things," IEEE Internet of Things Journal, vol. 8, no. 7, pp. 5350-5359, 2021.

[21] Z. Li, M. Zhu, Z. Huang et al., "Trajectory2Vec: a visual analytics approach for urban mobility patterns based on mobile phone data," Journal of Computer-Aided Design \& Computer Graphics, vol. 30, no. 1, pp. 68-70, 2018.

[22] F. Wang, W. Chen, Y. Zhao, T. Gu, S. Gao, and H. Bao, "Adaptively exploring population mobility patterns in flow visualization," IEEE Transactions on Intelligent Transportation Systems, vol. 18, no. 8, pp. 2250-2259, 2017.

[23] S. H. Zhang, D. D. Yin, Y. Q. Zhang et al., "Computing on base station behavior using Erlang measurement and call detail record," IEEE Transactions on Emerging Topics in Computing, vol. 3, no. 3, pp. 444-453, 2017.

[24] L. Chen, D. Yang, D. Zhang, C. Wang, J. Li, and T.-M.-T. Nguyen, "Deep mobile traffic forecast and complementary base station clustering for C-RAN optimization," Journal of Network and Computer Applications, vol. 121, no. 11, pp. 59-69, 2018.

[25] C. F. Liu, C. Liu, F. Q. Liu et al., "Clustering analysis of urban fabric detection based on mobile traffic data," Journal of Physics: Conference Series, vol. 1453, no. 1, pp. 152-158, 2020.

[26] Y. N. Peng, X. P. Fan, Y. Zhao et al., "A survey of visualization of temporal event sequence data," Journal of Computer-Aided Design \& Computer Graphics, vol. 5, no. 10, pp. 1698-1710, 2019.

[27] H. Y. Jang, Y. D. Wu, W. X. Zhao et al., "Mobile data visual analysis for human activity understanding," Journal of Zhejiang University (Science Edition), vol. 45, no. 1, pp. 40-46, 2018.

[28] H. Jiang, Y. Wu, Y. Zhang, S. Wang, and Y. Zhang, "From social community to spatio-temporal information: a new 
method for mobile data exploration," Journal of Visual Languages \& Computing, vol. 41, no. 8, pp. 1-9, 2017.

[29] F. Wang, B. R. Zhong, W. Yu et al., "Mutual inference model based on user roles and urban functional zones," Journal of Frontiers of Computer Science \& Technology, vol. 10, no. 12, pp. 1662-1672, 2016. 